Cite this: Soft Matter, 2014, 10, 4487

\title{
Magnetic control of flexoelectric domains in a nematic fluid
}

\author{
Péter Salamon, ${ }^{\text {aa }}$ Nándor Éber, ${ }^{a}$ Ágnes Buka, ${ }^{a}$ Tanya Ostapenko, ${ }^{b}$ Sarah Dölle ${ }^{b}$ \\ and Ralf Stannarius ${ }^{\mathrm{b}}$
}

The formation of flexoelectric stripe patterns (flexodomains) was studied under the influence of external electric and magnetic fields in a nematic liquid crystal. The critical voltage and wavevector of flexodomains were investigated in different geometries by both experiments and simulations. It is demonstrated that upon altering the orientation of the magnetic field with respect to the director, the critical voltage and wavenumber behave substantially differently. In the geometry of the twist Freedericksz transition, a non-monotonic behavior as a function of the magnetic field was found.

Received 22nd January 2014

Accepted 27th March 2014

DOI: $10.1039 / c 4 s m 00182 f$

www.rsc.org/softmatter field via the flexoelectric interaction. ${ }^{4,5}$ Flexoelectricity means that a polarization is induced by a splay or bend deformation of the director $\mathbf{n}$, and is defined as:

$$
\mathbf{P}_{\mathbf{f 1}}=e_{1} \mathbf{n}(\boldsymbol{\nabla n})+e_{3}(\boldsymbol{\nabla} \times \mathbf{n}) \times \mathbf{n},
$$
symmetry. ${ }^{1}$ They typically consist of elongated molecules that fluctuate around the local axis of symmetry described by a unit vector called the director (n). The practical importance of nematic liquid crystals originates from their controllability by external electric and magnetic fields. In display applications, an electric field is used to switch the director, which can adjust the optical properties of a device. ${ }^{2}$

The majority of display modes utilizes the Freedericksz transition: an external field-induced director reorientation, where the driving torques originate from the anisotropies of the dielectric constant $\left(\varepsilon_{\mathrm{a}}\right)$ and/or the diamagnetic susceptibility $\left(\chi_{\mathrm{a}}\right) .^{3}$ The value of $\varepsilon_{\mathrm{a}}\left(\chi_{\mathrm{a}}\right)$ is given by the difference of the dielectric constants (diamagnetic susceptibilities) measured in an electric field (magnetic field) parallel to and perpendicular to the director: $\varepsilon_{\mathrm{a}}=\varepsilon_{\|}-\varepsilon_{\perp}\left(\chi_{\mathrm{a}}=\chi_{\|}-\chi_{\perp}\right)$. If $\varepsilon_{\mathrm{a}}>0\left(\chi_{\mathrm{a}}>0\right)$, the director tends to be parallel to the applied electric (magnetic) field. Otherwise, the perpendicular configuration is more favorable. If a destabilizing field is precisely perpendicular to (or, for negative anisotropies, parallel to) the initial homogeneous director, the torque vanishes and the reorientation starts due to small fluctuations, above a well-defined threshold field.

In the electric Freedericksz transition, the dielectric interaction dominates, which is described by a free energy contribution quadratic in the magnitude of the electric field. In addition, the director may be coupled linearly with the electric

Institute for Solid State Physics and Optics, Wigner Research Centre for Physics, Hungarian Academy of Sciences, Budapest, Hungary. E-mail: salamon.peter@ wigner.mta.hu

${ }^{b}$ Institute for Experimental Physics, Otto-von-Guericke Universität, Universitätsplatz 2, 39106 Magdeburg, Germany where $e_{1}$ and $e_{3}$ are the splay and bend flexoelectric coefficients, respectively. The usual order of magnitude for $e_{1}$ and $e_{3}$ is $\mathrm{pC}$ $\mathrm{m}^{-1}$, though giant (a few $\mathrm{nC} \mathrm{m}^{-1}$ ) values ${ }^{6,7}$ were also reported for $e_{3}$ of bent-core ${ }^{8-14}$ liquid crystals.

Nematics are excellent materials to study spontaneous pattern formation, ${ }^{15}$ as nonlinearities in their physical properties provide a rich source of patterns, and external electromagnetic fields can serve as control parameters. For example, applying an electric field on a planar nematic layer can induce instabilities that result in different types of periodic director deformations.

In the present paper, we focus on a particular pattern, the socalled flexodomains (FDs), which represent an equilibrium director modulation caused by flexoelectricity. ${ }^{16,17}$ They appear as stripes parallel to the initial director $\mathbf{n}_{0}$. The first theoretical model of FDs only considered the one elastic constant approximation, ${ }^{16}$ but this has already given a good qualitative explanation of the phenomenon. Recently, a detailed theoretical description of FDs was developed ${ }^{18,19}$ that also accounted for unequal elastic constants and for the dynamic behavior of FDs exposed to sinusoidal voltage excitation. ${ }^{18}$ Furthermore, it recognized the similarity between FDs and splay-twist domains of the periodic Freedericksz transition; the latter were observed in polymeric liquid crystals with large elastic anisotropy ${ }^{20}$

Recently, nonlinear field effects and defect dynamics were also investigated in flexodomains ${ }^{21,22}$ in a bent-core compound. Moreover, flexoelectric patterns were studied in special geometries, such as twisted nematic (TN) cells using rod-like compounds $^{23}$ and recently in bent-core nematic liquid crystals,,$^{24,25}$ where the voltage-polarity dependent orientation of the 
flexoelectric stripes indicated that those domains are localized near the electrodes due to an electric field gradient.

In this work, we study how an additional magnetic field affects the formation of flexodomains. In order to give a complete answer, we performed experiments and developed a theoretical description, including magnetic fields applied in different geometries. Since flexodomains appear as an electric field-induced equilibrium deformation, similar to the electric Freedericksz transition, it is a plausible idea to compare the characteristics of these two phenomena in the presence of applied magnetic fields. In the present paper, we also make this comparison using our findings on flexodomains in magnetic fields.

The practical importance of flexodomains lies in the fact that they offer a method to determine the flexoelectric parameter $e^{*}=\left|e_{1}-e_{3}\right|$ that is otherwise only measurable by complicated or unreliable techniques. Classical measuring methods deduce flexoelectric parameters from the electro-optical response and require precise knowledge of the voltage applied on the liquid crystal. ${ }^{5}$ Since the director deformation originating from flexoelectricity is linearly coupled to the electric field, very low frequencies or DC voltages should be applied in order to avoid the damping of the optical response by the viscosities of the liquid crystal. Unfortunately, under such conditions, an internal voltage attenuation at the aligning layers and ionic effects $^{26-36}$ are unavoidable, resulting in erroneous voltage data. The main advantage of using FDs for determining $e^{*}$ is that the flexoelectric parameter can be calculated solely from the critical wavenumber, regardless of the value of the critical voltage. Indeed, analysis of FDs using the sophisticated theoretical description $^{18}$ has been successfully employed recently for measuring $e^{*}$ in a rod-like nematic. ${ }^{37}$ It should be noted, however, that the applicability of this method is limited; only a few compounds exhibit flexodomains, as the flexoelectric instability requires a special combination of material parameters. ${ }^{18}$ If the dielectric torque acting on the director is too large, the flexoelectric pattern formation is suppressed. Thus, an important requirement is a small $\left|\varepsilon_{\mathrm{a}}\right|$. We will show that the limits of applicability might be extended if a magnetic field is also applied.

\section{Experimental conditions}

Our experimental investigations were performed on a typical rod-like nematic liquid crystal 4- $n$-octyloxyphenyl 4- $n$-methyloxybenzoate $(1008 \dagger)$. The chemical structure of 1008 is shown in Fig. 1.

1008 shows only a nematic mesophase below the clearing point $\left(T_{\mathrm{NI}}\right)$ of $76.7^{\circ} \mathrm{C}$. On heating, it melts from the crystalline phase to the nematic phase at $63.5^{\circ} \mathrm{C}$; the nematic phase can be supercooled down to $53{ }^{\circ} \mathrm{C}$.

Several material parameters of 1008 were determined as a function of temperature in a previous work. ${ }^{37}$ Here, we will use the bulk elastic constants $\left(K_{11}, K_{22}\right.$, and $\left.K_{33}\right)$, the dielectric, and

$\dagger$ The same compound was abbreviated as $1 / 8$ by Kochowska et al. ${ }^{38}$ Here we rather follow an alternative nomenclature used by others. ${ }^{37,39,40}$

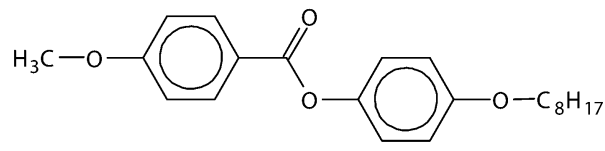

Fig. 1 The chemical structure of the rod-like molecule 4-n-octyloxyphenyl 4-n-methyloxybenzoate (1008).

the diamagnetic susceptibility anisotropies in our calculations. Our measurements were performed at $53{ }^{\circ} \mathrm{C}$, so we used the material parameters of 1008 corresponding to the same temperature in our simulations, namely: $K_{11}=8.54 \mathrm{pN}, K_{22}=$ $3.83 \mathrm{pN}, K_{33}=10.6 \mathrm{pN}, \varepsilon_{\mathrm{a}}=-0.48$, and $\chi_{\mathrm{a}}=9.65 \times 10^{-7}$.

The compound 1008 was studied in a sandwich cell with ITO electrodes coated with rubbed polyimide layers for planar alignment. The electrode area was $5 \mathrm{~mm} \times 5 \mathrm{~mm}$. The thickness of the empty cell $(d=19.5 \mu \mathrm{m})$ was measured by interferometry using an Ocean Optics spectrophotometer. During the experiments, the sample was held on a custom-made heat stage that provided a constant temperature with a precision better than $0.1{ }^{\circ} \mathrm{C}$. The heat stage was placed between the two poles of an electromagnet capable of producing a maximum homogeneous magnetic inductance of $B=1 \mathrm{~T}$ at the sample position. The magnetic inductance was measured by using an Alphalab 100 Gaussmeter. The magnetic field lay in the plane of the liquid crystal cell due to mechanical constraints. By rotating and fixing the stage, the angle between the magnetic field and the rubbing direction could be adjusted. Our measurements were performed in three geometries where this angle was set to $0^{\circ}, 45^{\circ}$, and $90^{\circ}$, henceforth denoted as the parallel $(\|)$, the oblique, and the perpendicular $(\perp)$ geometries, respectively (Fig. 2).

DC voltage $(U)$ was applied to the cell using the function generator output of a TiePie Handyscope HS3 device via a highvoltage amplifier. The sample was observed using a Questar QM100 long range microscope in transmission mode with white light illumination. The electric field-induced patterns were visualized by the shadowgraph technique, ${ }^{\mathbf{1 1}}$ without using any polarizer in the present case. The micrographs were recorded by using a Foculus FO323B digital camera.

In each geometry, for a given value of the magnetic field, voltage scans with $0.2 \mathrm{~V}$ steps were performed at a predefined voltage interval. After each voltage step, the DC driving was kept constant for 5 seconds before recording the image.

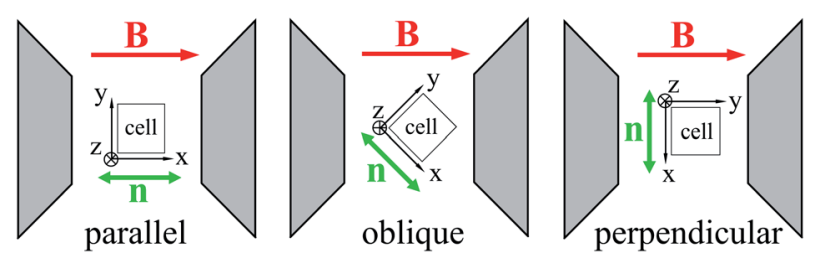

Fig. 2 The schematics of the measurement geometries referred to as parallel, oblique, and perpendicular. The plane of the sandwich cell lies in the plane of the figure ( $x-y$ plane), the observation direction and the electric field are parallel to the $z$-axis. 


\section{Theoretical model}

In order to understand the physics of flexoelectric pattern formation in the presence of the external magnetic field, one has to calculate the director distortions under the combination of electric and magnetic fields.

A planar cell filled with a nematic liquid crystal is considered in a three-dimensional Cartesian coordinate system. The $x$-axis coincides with the rubbing direction, and the cell lies in the $x-y$ plane. We assume strong anchoring of the director and no pretilt at the boundaries. The general director field $\mathbf{n}=\mathbf{n}(x, y, z)$ is represented by the tilt angle $\theta$ and the azimuthal (twist) angle $\phi$ :

$$
\mathbf{n}=(\cos \theta \cos \phi, \cos \theta \sin \phi, \sin \theta) .
$$

Then, the initial homogeneous orientation $\mathbf{n}_{0}$ corresponds to $\theta=\phi=0$, and both $\theta$ and $\phi$ should remain zero at the boundaries, even in the distorted state.

A homogeneous magnetic inductance $\mathbf{B}=\left(B_{\|}, B_{\perp}, 0\right)$ parallel to, and a homogeneous electric field perpendicular to the cell plane are considered. Naturally, the assumption on the homogeneity of the electric field is appropriate until the variation in the $z$-component of the director remains very small inside the cell, which is valid if $U \lesssim U_{\mathrm{c}}$.

$$
\mathbf{E}=\left(0,0, E_{z}\right)
$$

Since flexodomains represent an equilibrium deformation, the final state can be calculated by minimizing the free energy. In our case, the density of free energy $(f)$ is given by the sum of the elastic $\left(f_{\text {elast }}\right)$, dielectric $\left(f_{\text {electr }}\right)$, flexoelectric $\left(f_{\text {flexo }}\right)$, and magnetic $\left(f_{\text {magn }}\right)$ contributions:

$$
\begin{gathered}
f=f_{\text {elast }}+f_{\text {diel }}+f_{\text {flexo }}+f_{\text {magn }} \\
f_{\text {elast }}=\frac{1}{2} K_{11}(\nabla \mathbf{n})^{2}+\frac{1}{2} K_{22}(\mathbf{n}(\nabla \times \mathbf{n}))^{2}+\frac{1}{2} K_{33}(\mathbf{n} \times(\nabla \times \mathbf{n}))^{2} \\
f_{\text {diel }}=-\frac{1}{2} \varepsilon_{0} \varepsilon_{\mathrm{a}}(\mathbf{n} \mathbf{E})^{2} \\
f_{\text {flexo }}=-e_{1} \mathbf{n} \mathbf{E}(\nabla \mathbf{n})+e_{3}(\mathbf{n} \times(\nabla \times \mathbf{n})) \mathbf{E} \\
f_{\text {magn }}=-\frac{1}{2} \frac{\chi_{\mathrm{a}}}{\mu_{0}}(\mathbf{n B})^{2} .
\end{gathered}
$$

The Frank elastic constants $K_{11}, K_{22}$, and $K_{33}$ correspond to the splay, twist, and bend director deformations, respectively. The permittivity and permeability of vacuum are denoted by $\varepsilon_{0}$ and $\mu_{0}$, respectively. For the minimization of the free energy the Euler-Lagrange formalism is used.

The characteristic parameters of the flexodomains, namely their threshold voltage $U_{\mathrm{c}}$ and the critical wavevector $\mathbf{q}_{\mathrm{c}}$ at the onset of the flexoelectric instability, can be obtained via a linear stability analysis with respect to periodic director deformations. These detailed calculations will be performed below for two special cases, the parallel and the perpendicular geometries shown in Fig. 2.

\subsection{The parallel geometry}

In the parallel geometry, the magnetic inductance is

$$
\mathbf{B}=\left(B_{\|}, 0,0\right) .
$$

Assuming $\chi_{\mathrm{a}}>0$, no magnetic Freedericksz transition is expected in this geometry; thus, the modulated director field of flexodomains emerges from a homogeneous planar basic state. The stripes of FDs are assumed to remain parallel to the rubbing direction, $\mathbf{q}_{\mathrm{c}}=(0, q, 0)$. Consequently, all variables depend only on the $y$ - and $z$-coordinates. The free energy is minimized by solving the system of Euler-Lagrange equations:

$$
\begin{aligned}
& \frac{\mathrm{d}}{\mathrm{d} y}\left(\frac{\partial f}{\partial \theta_{, y}}\right)+\frac{\mathrm{d}}{\mathrm{d} z}\left(\frac{\partial f}{\partial \theta_{, z}}\right)-\frac{\partial f}{\partial \theta}=0, \\
& \frac{\mathrm{d}}{\mathrm{d} y}\left(\frac{\partial f}{\partial \phi_{, y}}\right)+\frac{\mathrm{d}}{\mathrm{d} z}\left(\frac{\partial f}{\partial \phi_{, z}}\right)-\frac{\partial f}{\partial \phi}=0,
\end{aligned}
$$

where spatial partial derivatives are denoted in the lower indices by commas and the corresponding space coordinates.

Combining eqn (2)-(11) results in a complicated system of nonlinear partial differential equations that has to be further processed as follows. Near the onset of flexodomains, the director distortions are small and their periodic part characterized by the wavenumber $q$ can be separated from the $z$ dependent amplitudes of the tilt $\left(\theta_{0}(z)\right)$ and twist $\left(\phi_{0}(z)\right)$ modulations via:

$$
\begin{aligned}
& \theta(y, z)=\theta_{0}(z) \cos (q y), \\
& \phi(y, z)=\phi_{0}(z) \sin (q y) .
\end{aligned}
$$

The director deformation profile of FDs in the middle of the cell $(z=0)$ is shown in Fig. 3. Using the above ansatz, eqn (10) and (11) can be linearized with respect to the small quantities $\theta_{0}$ and $\phi_{0}$. After switching to the dimensionless space variable $\hat{z}=$ $z \pi / d$ and wavenumber $\hat{q}=q d / \pi$, straightforward calculations result in:

$$
\begin{aligned}
\phi_{0}^{\prime \prime}= & 2 \frac{\delta K \hat{q}}{1-\delta K} \theta_{0}^{\prime}-\frac{U e^{*} \hat{q}}{K_{\mathrm{av}}(1-\delta K) \pi} \theta_{0} \\
& +\left(\left(\frac{B_{\|}}{B_{\mathrm{t}}}\right)^{2}+\frac{(1+\delta K) \hat{q}^{2}}{1-\delta K}\right) \phi_{0},
\end{aligned}
$$
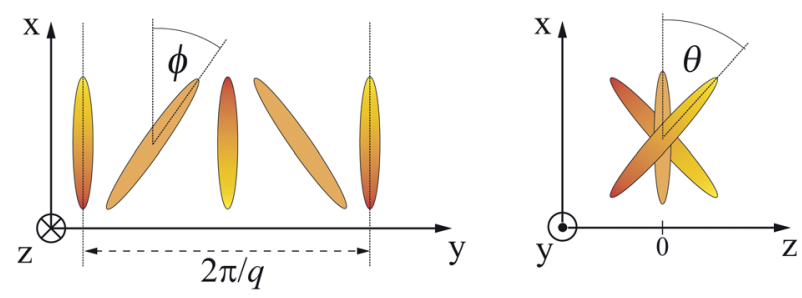

Fig. 3 The director profile of flexodomains in the middle of the cell $(z=0)$ in two views. The director is symbolized by ellipses. The electric field is parallel to the $z$-direction. 


$$
\begin{aligned}
\theta_{0}^{\prime \prime}= & -2 \frac{\delta K \hat{q}}{1+\delta K} \phi_{0}^{\prime}-\frac{U e^{*} \hat{q}}{K_{\mathrm{av}}(1+\delta K) \pi} \phi_{0} \\
& +\left(\left(\frac{B_{\|}}{B_{\mathrm{s}}}\right)^{2}-\left(\frac{U}{U_{\mathrm{s}}}\right)^{2}+\frac{(1-\delta K) \hat{q}^{2}}{1+\delta K}\right) \theta_{0}
\end{aligned}
$$

where $K_{\mathrm{av}}=\left(K_{11}+K_{22}\right) / 2$, and $\delta K=\left(K_{11}-K_{22}\right) /\left(K_{11}+K_{22}\right)$. In this final system of ordinary differential equations, the prime and double prime denote the first and second $\hat{z}$-derivatives, respectively. In addition, the following scaling quantities are introduced: $B_{\mathrm{s}}=\frac{\pi}{d} \sqrt{\frac{\mu_{0} K_{11}}{\chi_{\mathrm{a}}}}, B_{\mathrm{t}}=\frac{\pi}{d} \sqrt{\frac{\mu_{0} K_{22}}{\chi_{\mathrm{a}}}}$, and $U_{\mathrm{s}}=\pi \sqrt{\frac{K_{11}}{\varepsilon_{0} \varepsilon_{\mathrm{a}}}}$. They are formally similar to the expressions for the threshold magnetic inductances of the magnetic splay $\left(B_{\mathrm{S}}\right)$, the magnetic twist $\left(B_{\mathrm{t}}\right)$ transitions, and the threshold voltage $U_{\mathrm{s}}$ of the electric splay Freedericksz transition. The applied voltage corresponds to $U=E_{z} d$.

The cell is symmetric with respect to its midplane, therefore it is sufficient to perform the calculations for only one half of the cell. For a given value of $\hat{q}$ and $U$, the system of eqn (14) and (15) was numerically solved for $\phi_{0}(\hat{z})$ and $\theta_{0}(\hat{z})$ in Matlab in the interval $\hat{z}=[-\pi / 2,0]$, which corresponds to the lower half of the planar cell. Mixed boundary conditions were used as follows: $\theta_{0}(-\pi / 2)=\phi_{0}(-\pi / 2)=0$ and $\theta_{0}^{\prime}(0)=\phi_{0}^{\prime}(0)=0$.

For $U<U_{\mathrm{c} \| \hat{q}}$ the homogeneous director field is stable, thus $\phi_{0}(\hat{z})=\theta_{0}(\hat{z})=0$. The critical voltage $U_{\mathrm{c} \| \hat{q}}$ is identified by the nonzero solutions of the director modulation amplitudes $\phi_{0}(\hat{z})$ and $\theta_{0}(\hat{z})$, showing the emergence of the pattern. Since our model is linearized, no quantitative information can be obtained about the director field above the critical voltage.

Calculating $U_{\mathrm{c} \| \hat{q}}$ as the function of $\hat{q}$ yields a neutral curve with a minimum corresponding to the actual critical voltage $U_{\mathrm{c} \|}$ and wavenumber $\hat{q}_{\mathrm{c} \|}$. As an example, the $U_{\mathrm{c} \| \hat{q}} v s$. $\hat{q}$ curve is shown for $B_{\|}=0 \mathrm{~T}$ (solid line), $0.25 \mathrm{~T}$ (dotted line), $0.375 \mathrm{~T}$ (dashed line), and $0.5 \mathrm{~T}$ (dash-dotted line) in Fig. 4. The red crosses show $U_{\mathrm{c} \|}$ and $\hat{q}_{\mathrm{c} \|}$ for each value of the applied parallel magnetic inductance. The calculations were performed with the material parameters of 1008 listed in Section 2 and $e^{*}=$ $6.9 \mathrm{pC} \mathrm{m}^{-1}$.

It can be clearly seen in Fig. 4 that both $U_{\mathrm{c} \|}$ and $\hat{q}_{\mathrm{c} \|}$ increase with higher values of $B_{\|}$.

\subsection{The perpendicular geometry}

In the perpendicular geometry, the magnetic inductance is given by:

$$
\mathbf{B}=\left(0, B_{\perp}, 0\right)
$$

The main difference to the parallel case is that here the magnetic field does not stabilize the initial homogeneous planar configuration; instead it induces a twist Freedericksz transition.

As a consequence, in the absence of the electric field, the director can be described solely by a $\hat{z}$-dependent twist angle $\psi=\psi(\hat{z})$, i.e. $\mathbf{n}=(\cos \psi, \sin \psi, 0)$. The determination of the director profile via minimization of the free energy is a

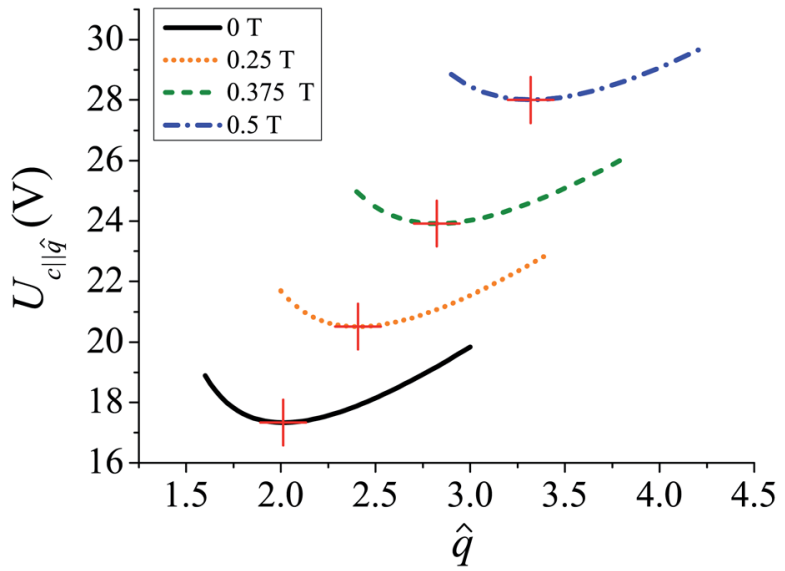

Fig. 4 The critical voltages $\left(U_{c \| \hat{q}}\right)$ of flexodomains for different wavenumbers in the case of $B_{\|}=0 \mathrm{~T}$ (solid line), $0.25 \mathrm{~T}$ (dotted line), $0.375 \mathrm{~T}$ (dashed line), and $0.5 \mathrm{~T}$ (dash-dotted line). For a given magnetic inductance, the red cross shows the smallest critical voltage $U_{c\|\|}$ at the critical wavenumber $\hat{q}_{c \| \mid}$ that should actually be realized by the system.

well-known procedure; $\psi(\hat{z})$ can be obtained as the solution of the second order nonlinear ordinary differential equation: ${ }^{\mathbf{4 2}}$

$$
\psi^{\prime \prime}=-\left(\frac{B_{\perp}}{B_{\mathrm{t}}}\right)^{2} \cos \psi \sin \psi
$$

with the boundary conditions: $\psi_{0}(-\pi / 2)=0$ and $\psi_{0}^{\prime}(0)=0$.

Fig. 5 shows the resulting $\psi(\hat{z})$ profile inside the cell calculated with the parameters of our particular material (10O8) for three different values of the applied magnetic inductance.

For $\mathrm{B}_{\perp}<B_{\mathrm{t}}$, the twist angle naturally equals zero. Above the Freedericksz threshold field, $\psi$ increases and reaches its maximum in the middle of the cell: $\psi_{\mathrm{m}}=\psi(0)$. At higher $B_{\perp}, \psi_{\mathrm{m}}$ approaches $90^{\circ}$, but in the largest part of the cell, the twist angle is still significantly below $90^{\circ}$; even at $B_{\perp} / B_{\mathrm{t}}=2.78$ that corresponds to our maximum inductance of $B_{\perp}=1 \mathrm{~T}$. Note that an electric field below the onset of FDs (i.e. $\left.U<U_{\mathrm{c} \|}\right)$ does not affect the basic (homogeneous or twisted) state.

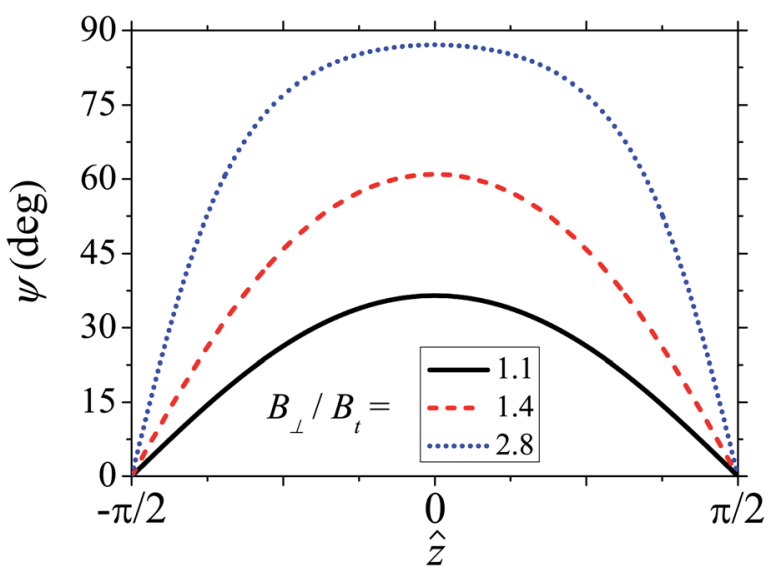

Fig. 5 The twist angle $\psi$ versus the cross-section direction $z$ of the planar cell in the case of different perpendicularly applied magnetic inductances. 
If $B_{\perp}<B_{\mathrm{t}}$, the initial director configuration is homogeneous, thus the director modulation caused by the onset of flexodomains can be described similar to the parallel case, using eqn (2). Above the Freedericksz threshold, however, the periodic structure of FDs emerges from a twisted director field, hence:

$$
\mathbf{n}=(\cos \theta \cos (\psi+\phi), \cos \theta \sin (\psi+\phi), \sin \theta),
$$

where $\theta=\theta(x, y, z)$ and $\phi=\phi(x, y, z)$ now depend on all space coordinates. The free energy minimization can be done by the system of Euler-Lagrange equations:

$$
\begin{gathered}
\frac{\mathrm{d}}{\mathrm{d} x}\left(\frac{\partial f}{\partial \theta_{, x}}\right)+\frac{\mathrm{d}}{\mathrm{d} y}\left(\frac{\partial f}{\partial \theta_{, y}}\right)+\frac{\mathrm{d}}{\mathrm{d} z}\left(\frac{\partial f}{\partial \theta_{, z}}\right)-\frac{\partial f}{\partial \theta}=0, \\
\frac{\mathrm{d}}{\mathrm{d} x}\left(\frac{\partial f}{\partial \phi_{, x}}\right)+\frac{\mathrm{d}}{\mathrm{d} y}\left(\frac{\partial f}{\partial \phi_{, y}}\right)+\frac{\mathrm{d}}{\mathrm{d} z}\left(\frac{\partial f}{\partial \phi_{, z}}\right)-\frac{\partial f}{\partial \phi}=0 .
\end{gathered}
$$

In eqn (19) and (20), additional terms appear compared to eqn (10) and (11) in the parallel case, due to the $x$-dependence of the angles $\theta$ and $\phi$. The combination of eqn (3)-(8) and (16)-(20) leads to lengthy expressions that must be linearized next in order to have a chance to calculate the threshold parameters of FDs.

In the perpendicular geometry we still assume that the flexoelectric instability results in unidirectional stripes, but in contrast to the parallel case, the stripes are allowed to run at an angle $\beta$ with respect to the initial planar director $\mathbf{n}_{0}$, i.e. $\mathbf{q}_{\mathbf{c}}=$ ( $q \sin \beta, q \cos \beta, 0)$. Hence the following ansatz is applied to the $\theta$ and $\phi$ angles:

$$
\begin{aligned}
& \theta(x, y, z)=\theta_{0}(z) \cos ((q \cos \beta) y-(q \sin \beta) x), \\
& \phi(x, y, z)=\phi_{0}(z) \sin ((q \cos \beta) y-(q \sin \beta) x) .
\end{aligned}
$$

By switching again to dimensionless variables as in Section 3.1 after straightforward calculations one obtains the system of ordinary differential equations:

$$
\begin{aligned}
\phi_{0}^{\prime \prime}= & \hat{q} \theta_{0}\left(\left(1-\frac{\kappa}{1-\delta K}\right) \psi^{\prime} \sin (\beta-\psi)-\frac{e^{*} U \cos (\beta-\psi)}{(1-\delta K) K_{a v} \pi}\right) \\
& +\phi_{0}\left(\frac{\kappa \hat{q}^{2} \sin ^{2}(\beta-\psi)}{1-\delta K}+\frac{(1+\delta K) \hat{q}^{2} \cos ^{2}(\beta-\psi)}{1-\delta K}\right. \\
& \left.-\left(\frac{B_{\perp}}{B_{t}}\right)^{2} \cos (2 \psi)\right)+\frac{2 \delta K \hat{q} \theta_{0}^{\prime} \cos (\beta-\psi)}{1-\delta K} \\
\theta_{0}^{\prime \prime}= & \hat{q} \phi_{0}\left(\frac{(1-3 \delta K-\kappa) \psi^{\prime} \sin (\beta-\psi)}{1+\delta K}-\frac{e^{*} U \cos (\beta-\psi)}{(1+\delta K) K_{a v} \pi}\right) \\
& +\theta_{0}\left(\frac{\kappa \hat{q}^{2} \sin ^{2}(\beta-\psi)}{1+\delta K}+\frac{(1-\delta K) \hat{q}^{2} \cos ^{2}(\beta-\psi)}{1+\delta K}\right. \\
& \left.+\frac{(2 \delta K+\kappa-2)\left(\psi^{\prime}\right)^{2}}{1+\delta K}+\left(\frac{B_{\perp}}{B_{\mathrm{s}}}\right)^{2} \sin ^{2}(\psi)-\left(\frac{U}{U_{\mathrm{s}}}\right)^{2}\right) \\
& -\frac{2 \delta K \hat{q} \phi_{0}^{\prime} \cos (\beta-\psi)}{1+\delta K},
\end{aligned}
$$

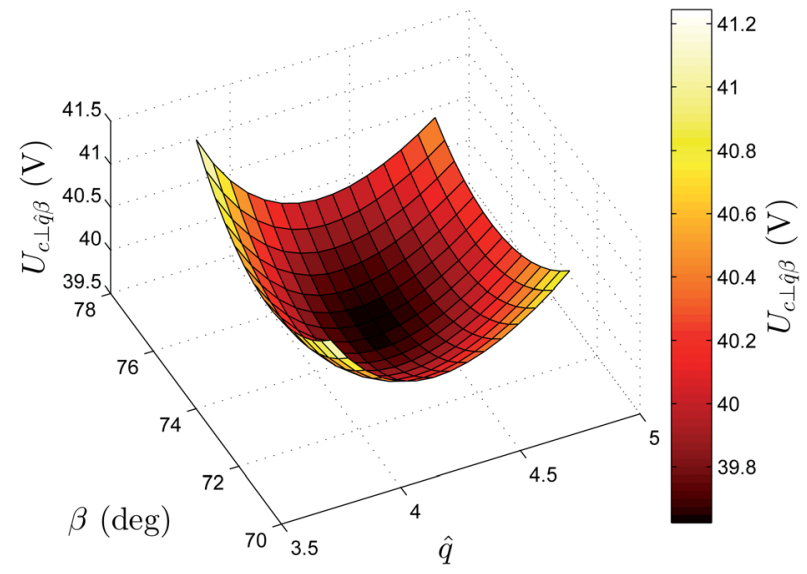

Fig. 6 The critical voltages $\left(U_{c \perp \hat{q} \beta}\right)$ of flexodomains for different wavenumbers $\hat{q}$ and stripe angles $\beta$ in the case of $B_{\perp}=0.7 \mathrm{~T}$. The minimum of the surface in the center corresponds to the actual threshold of the flexoelectric instability.

where an additional constant $\kappa=K_{33} / K_{\mathrm{av}}$ was introduced.

Below the twist Freedericksz threshold, the procedure to find $U_{\mathrm{c} \perp}$ and $\hat{q}_{\mathrm{c} \perp}$ for different values of $B_{\perp}$ is similar to that discussed in Section 3.1, as $\beta$ and $\psi$ can be fixed to zero. If $B_{\perp}>B_{\mathrm{t}}$, however, $\psi$ and $\psi^{\prime}$ have to be taken from the solution of eqn (17). Calculating $U_{\mathrm{c} \perp \hat{q} \beta}$ as the function of $\hat{q}$ and $\beta$ gives a surface with a minimum that corresponds to the actual critical voltage $U_{\mathrm{c} \perp}$, wavenumber $\hat{q}_{\mathrm{c} \perp}$, and stripe angle $\beta_{\mathrm{c} \perp}$.

As an example, $U_{\mathrm{c} \perp \hat{q} \beta}$ plotted as the function of $\hat{q}$ and $\beta$ for $B_{\perp}=0.7 \mathrm{~T}$ is shown in Fig. 6 . The calculations were performed using the material parameters of 1008 presented in Section 2 and $e^{*}=6.8 \mathrm{pC} \mathrm{m}^{-1}$. The minimum is clearly seen at around the middle of the surface.

\section{Experimental results}

\subsection{Parallel geometry}

Snapshots of flexodomains taken at $B_{\|}=0 \mathrm{~T}(U=23 \mathrm{~V})$ and $B_{\|}=$ $1 \mathrm{~T}(U=52.6 \mathrm{~V})$ are presented in Fig. 7a and c. The micrographs were captured slightly above the threshold voltages $\left(U_{\mathrm{c} \|}\right)$ of the patterns in the parallel geometry, covering an area of $106 \mu \mathrm{m} \times$ $106 \mu \mathrm{m}$. The two-dimensional Fourier transforms (amplitude spectra) of the images Fig. 7a and c are shown in Fig. 7b and d, respectively.

It can immediately be seen in Fig. 7 that the dimensionless wavenumber $\hat{q}$ of FDs is significantly larger at $B_{\|}=1 \mathrm{~T}$ than at zero magnetic field, however, the direction of the wavevector remains the same as expected. The threshold voltage is also larger at the higher $B_{\|}$. In order to precisely determine the threshold parameters $U_{\mathrm{c} \|}$ and $\hat{q}_{\mathrm{c} \|}$, the emergence of the pattern has to be followed as the function of the applied voltage. The proper analysis of this process needs a definition of a quantity that can be used to indicate the presence of a pattern. In our case, this quantity was assigned to the maximal Fourier amplitude $\left(C_{\mathrm{FFT}}\right)$ in a broad region in that part of the Fourier space where the peaks for FDs were expected. The value of $C_{\mathrm{FFT}}$ is essentially a measure of contrast that is expected to be 
(a)

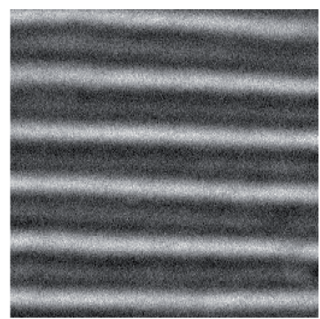

(b)

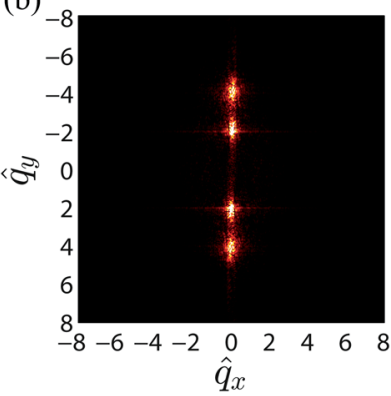

(c)

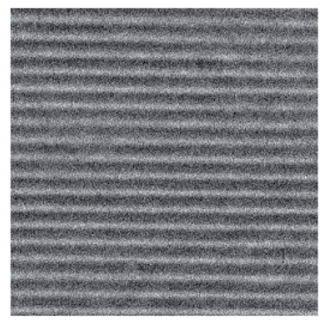

(d)

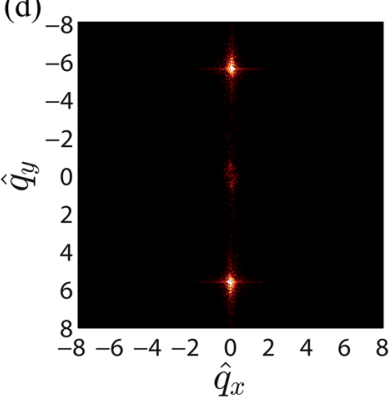

Fig. 7 Micrographs of flexodomains taken at (a) $B_{\|}=0 \mathrm{~T}(U=23 \mathrm{~V})$ and (c) $B_{\|}=1 \mathrm{~T}(U=52.6 \mathrm{~V})$ in the parallel geometry. The images cover an area of $106 \mu \mathrm{m} \times 106 \mu \mathrm{m}$. The magnetic field and the rubbing direction lie parallel to the horizontal direction. The two dimensional Fourier transforms of (a) and (c) are shown in (b) and (d), respectively.

minimal in the homogeneous initial state, and to increase with the emergence of the pattern.

The measured voltage dependence of $C_{\mathrm{FFT}}$ is shown in Fig. 8 for different values of $B_{\|}$. We note that here $C_{\mathrm{FFT}}$ is background corrected, which means that the maximal Fourier amplitude of the snapshot taken in the homogeneous initial state is subtracted from all measured values.

The threshold behavior is observed from Fig. 8 for all different values of $B_{\|}$. Below the appearance of the patterns, the contrast equals the background value, thus $C_{\mathrm{FFT}}=0$. At higher voltages, the emergence of FDs is indicated by an increase in the contrast. The critical voltages $U_{\mathrm{c} \|}$ (versus $B_{\|}$) were determined by extrapolation of the lines fitted on the linear parts of the $C_{\mathrm{FFT}}(U)$ functions for each value of $B_{\|}$(dashed lines in Fig. 8).

The $\hat{q}$ data were obtained by fitting the peaks in the Fourier transforms of micrographs with 2D Gaussian surfaces for each applied voltage. The fitted centres of the Gaussians were used to acquire the values of $\hat{q}$. In Fig. 9, the wavenumbers of FDs are plotted as the function of the reduced voltage $U / U_{\mathrm{c} \|}$ for several values of $B_{\|}$. The data show that the wavenumber increases linearly with the applied voltage above the threshold. Therefore, the critical wavenumber $\hat{q}_{\mathrm{c} \|}$ should be determined by extrapolation to $U / U_{\mathrm{c} \|}=1$. The extrapolating dashed lines in Fig. 9 were fitted to the data points lying in the range $1.02<U / U_{\mathrm{c} \|}<1.06$, which is approximately the same interval as the one used in the extrapolation to determine $U_{\mathrm{c} \|}$ (see Fig. 8).

Applying the procedure presented above on a number of different $B_{\|}$values, the magnetic field dependence of the threshold parameters can be determined. Fig. 10a and b depict how $B_{\|}$affects $U_{\mathrm{c} \|}$ and $\hat{q}_{\mathrm{c} \|}$, respectively. The solid symbols show the experimentally obtained data.

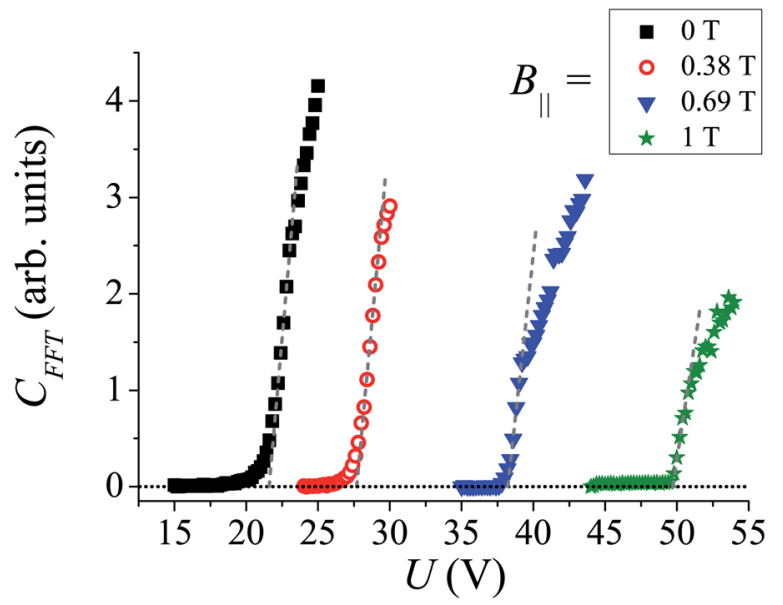

Fig. 8 The voltage dependence of the pattern contrast (symbols) based on the maximal Fourier amplitude $\left(C_{\mathrm{FFT}}\right)$ for different applied magnetic inductances in the parallel geometry. The dashed lines indicate the linear extrapolation.

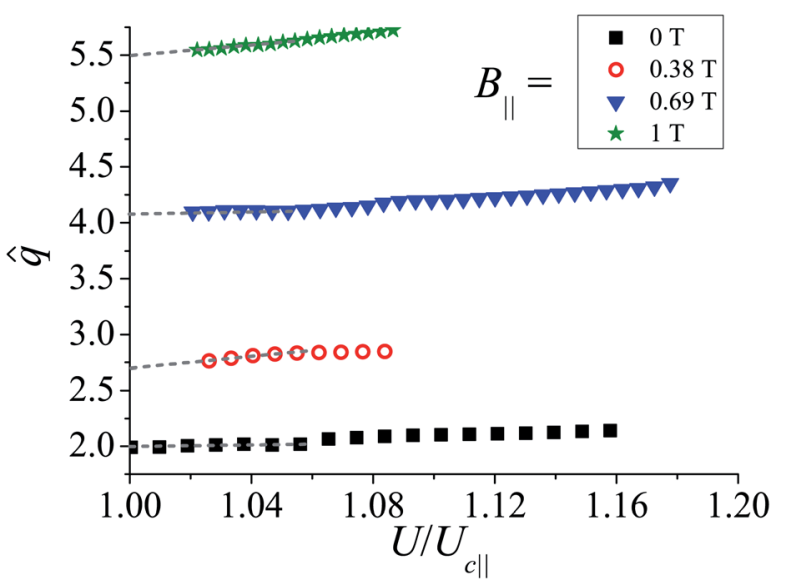

Fig. 9 The wavenumber of flexodomains as a function of the reduced voltage (symbols) for different applied magnetic inductances in the parallel geometry. The dashed lines indicate the linear extrapolation.

In order to see how the experimental results match with our theoretical considerations, the threshold parameters $U_{\mathrm{c} \|}$ and $\hat{q}_{\mathrm{c} \|}$ were determined by the simulation technique described in Section 3.1 using the material parameters of 1008 listed in Section 2. Only the parameter $e^{*}$, i.e. the difference of flexoelectric coefficients, was determined by fitting our theoretical model to the measured value of $\hat{q}_{\mathrm{c} \|}$ at $B_{\|}=0$. Our method gave $e^{*}=6.9 \mathrm{pC} \mathrm{m}^{-1}$, which was used in the simulations of the parallel geometry. The open symbols in Fig. 10a and b show the magnetic inductance dependence of the critical voltage and the wavenumber obtained from the simulation (the connecting lines are used as guides for the eye).

It is seen in Fig. 10 that the theoretical $U_{\mathrm{c} \|}(B)$ dependence is nicely reproduced experimentally; $U_{\mathrm{c} \|}$ increases monotonically with $B_{\|}$, but the measured threshold voltages are systematically larger than the theoretical ones. This deviation can be attributed to the ionic conductivity of the liquid crystal and to the 

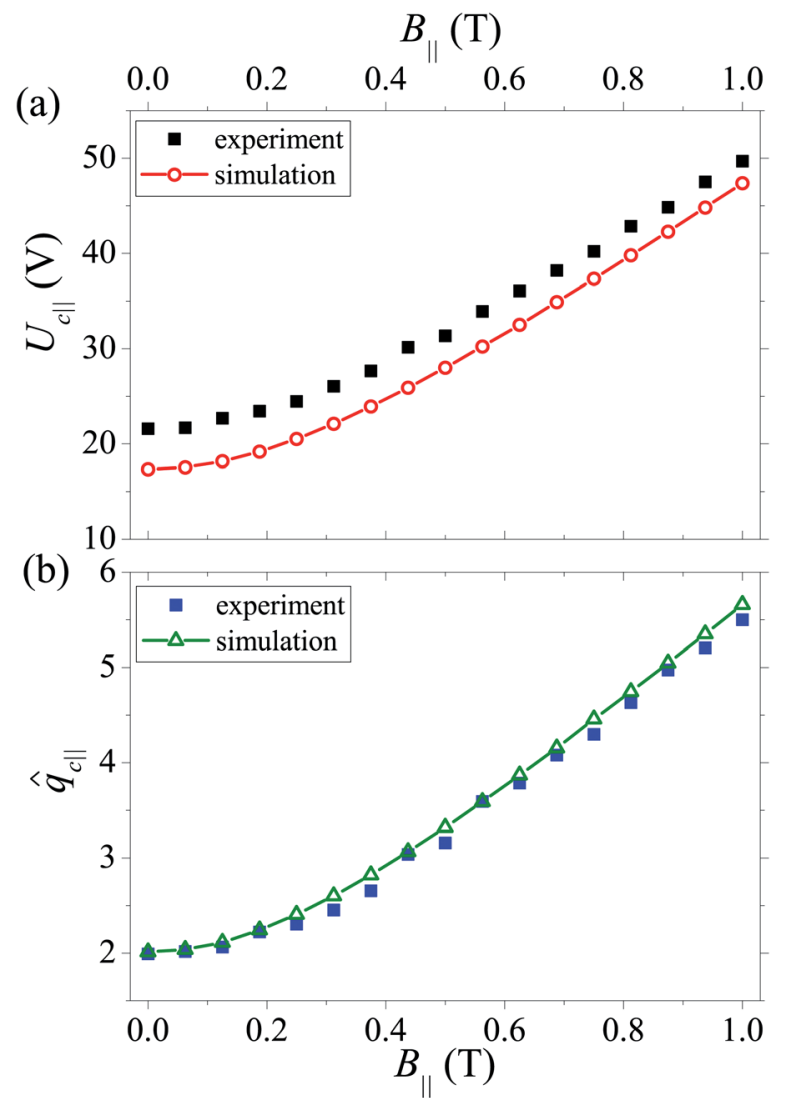

Fig. 10 The magnetic inductance dependence of (a) the critical voltage and (b) the wavenumber in the parallel geometry. The solid (connected open) symbols were obtained by experiments (by numerical simulations).

structure of the cell. Though in many situations liquid crystals can be considered to be insulators, the finite conductivity of nematics becomes important if a low frequency AC voltage is applied onto the material. ${ }^{43}$ The effect is even more apparent when a DC voltage is used. Common liquid crystals, such as 1008, exhibit electrolytic conductivity where the charge carriers are ionic impurities. If the applied electric field changes very slowly or is constant with time, ions with opposite charges have time to reach the opposite electrodes, where they can accumulate, forming a Debye layer. This screening may reduce the electric field in the cell. However, if the total number of charge carriers is sufficiently low, this effect is negligible. In typical liquid crystal test cells, the ITO electrodes are coated with electrically insulating polyimide layers. The thickness of these are approximately 100$120 \mathrm{~nm}$ that can provide barriers strong enough to stop ions and minimize the charge transfer from the electrodes..$^{28}$ In the static case, the voltage $U$ applied on the cell should be larger than that on the liquid crystal itself $\left(U_{\mathrm{LC}}\right)$, because of the voltage drop at the polyimide and the Debye layers. The internal voltage attenuation may be estimated by $U_{\mathrm{LC}} / U=R_{\mathrm{LC}} /\left(R_{\mathrm{b}}+R_{\mathrm{LC}}\right)$, where $R_{\mathrm{LC}}$ and $R_{\mathrm{b}}$ are the resistances of the liquid crystal and of the boundary layers, respectively. This simple model can explain why systematically larger critical voltages were obtained in the experiments compared to the simulations.
Another peculiarity seen in Fig. 10a is that the difference between the experimental and calculated critical voltages is larger at lower applied voltages. This effect is consistent with the above model. Increasing the applied DC voltage decreases the effective number of charge carriers and thus increases $R_{\mathrm{LC}}$, while $R_{\mathrm{p}}$ might be regarded as voltage independent. Consequently, the internal attenuation reduces; the ratio $U_{\mathrm{LC}} / U$ approaches 1 when the applied voltage increases.

Besides the critical voltage, the wavenumber $\hat{q}_{\mathrm{c} \|}$ also shows a significant increase with the applied magnetic field (see Fig. 7 and 10). This tendency is the consequence of the monotonically increasing $U_{\mathrm{c} \|}\left(B_{\|}\right)$; higher voltages may allow higher wavenumbers. The simulation results agree very well with the experimental data. It should be noted that the calculated magnetic field dependence of $\hat{q}_{\mathrm{c} \|}$ in Fig. 10b is not a fit; the material parameters used were not varied in order to achieve a better match with the measured results. This implies that a fine tuning of the elastic constants and the other parameters may result in even better agreement.

\subsection{The oblique geometry}

In Fig. 11a-d, micrographs of FDs of the oblique geometry that were taken at $B_{\text {obl }}=0 \mathrm{~T}(U=23 \mathrm{~V}), B_{\text {obl }}=0.3 \mathrm{~T}(U=27.6 \mathrm{~V}), B_{\text {obl }}$ $=0.6 \mathrm{~T}(U=38.8 \mathrm{~V})$ and $B_{\mathrm{obl}}=1 \mathrm{~T}(U=54.8 \mathrm{~V})$, respectively, are shown. The images were recorded slightly above the threshold voltages of flexodomains, and they cover an area of $106 \mu \mathrm{m} \times$ $106 \mu \mathrm{m}$, similar to the ones presented in the previous subsection of the parallel geometry. In Fig. 11a-d, one can clearly identify the most spectacular feature of the oblique geometry: not only the wavenumber of the FD stripes is influenced by the magnetic field, but the direction of the wavevector as well.

In the oblique geometry, the threshold voltages $U_{\text {cobl }}$ and the critical wavenumbers $\hat{q}_{\text {cobl }}$ were determined following the procedure presented in Section 4.1 in conjunction with Fig. 8 and 9. In contrast to the parallel case, the angle $\beta_{\text {obl }}$ between $\mathbf{n}_{0}$ and the flexoelectric stripes had to be measured too.

The dependence of the stripe direction $\beta_{\text {obl }}$ on the reduced voltage $U / U_{\text {cobl }}$ is plotted in Fig. 12 for different magnetic inductances. At nonzero values of $B_{\mathrm{obl}}$, the angle $\beta_{\mathrm{obl}}$ shows a decreasing tendency by increasing $U / U_{\text {cobl. }}$ Therefore the stripe direction angle at the onset of the flexoelectric patterns $\left(\beta_{\text {cobl }}\right)$ can be determined by extrapolation (see the dashed lines in Fig. 12), analogous to the determination of $\hat{q}_{\text {cobl }}$.

The magnetic inductance dependence of the threshold parameters $U_{\text {cobl }}$ and $\hat{q}_{\text {cobl }}$ is shown in Fig. 13a. The critical voltage increases with $B_{\text {obl }}$; $\hat{q}_{\text {cobl }}$ exhibits a similar character. The tendency of increasing the critical voltage and wavenumber at high magnetic fields is similar to that found in the parallel geometry and is due to the stabilizing magnetic torques.

The stripe angle $\beta_{\text {cobl }}$ at the threshold versus the magnetic inductance is shown in Fig. 13b. The data indicate that $\beta_{\text {cobl }}$ increases with $B_{\mathrm{obl}}$ monotonically from zero and it approaches $45^{\circ}$ at high fields.

In the oblique geometry, a pure magnetic field induces a thresholdless, homogeneous twist deformation. Therefore, for $B_{\text {obl }} \neq 0$, a twisted structure forms the basic state of the 
(a)

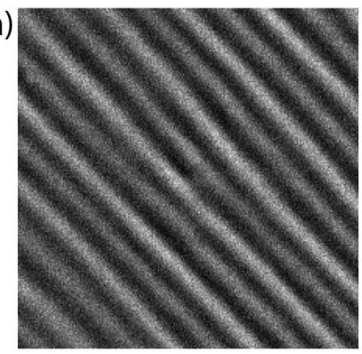

(c)

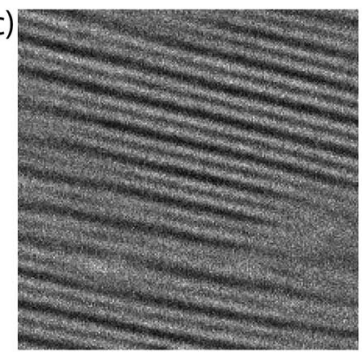

(b)

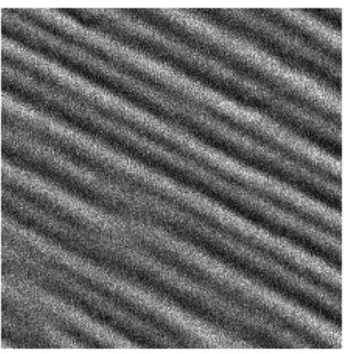

(d)

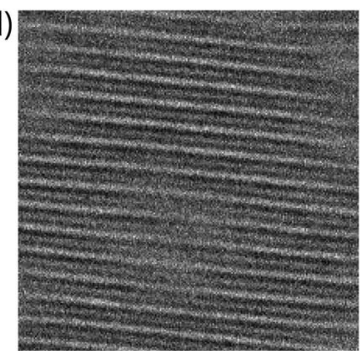

Fig. 11 Micrographs of flexodomains taken at (a) $B_{\mathrm{obl}}=0 \mathrm{~T}(U=23 \mathrm{~V})$, (b) $B_{\mathrm{obl}}=0.3 \mathrm{~T}(U=27.6 \mathrm{~V})$, (c) $B_{\mathrm{obl}}=0.6 \mathrm{~T}(U=38.8 \mathrm{~V})$ and (d) $B_{\mathrm{obl}}=1$ $\mathrm{T}(U=54.8 \mathrm{~V})$ in the oblique geometry. The images cover an area of $106 \mu \mathrm{m} \times 106 \mu \mathrm{m}$. The magnetic field lies in the horizontal direction. The rubbing direction is at an angle of $45^{\circ}$ with respect to the horizontal direction (parallel to the stripes in (a)).

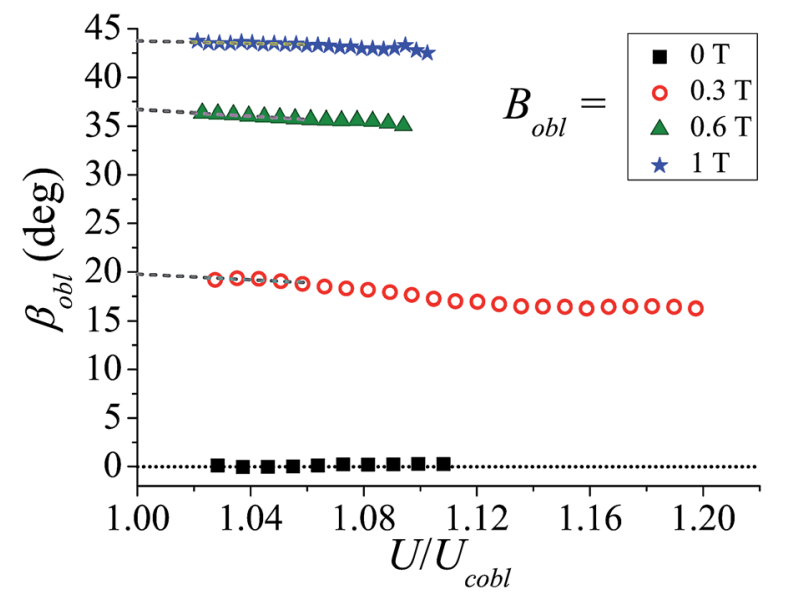

Fig. 12 The angle of FD stripes with respect to the rubbing direction as the function of the reduced voltage (symbols) for different applied magnetic inductances in the oblique geometry. The dashed lines indicate the linear extrapolation.

flexoelectric instability at $U=U_{\text {cobl. }}$ For high magnetic fields, the director realigns to be parallel to $\mathbf{B}_{\mathrm{obl}}$, i.e. the maximal rotation angle is $45^{\circ}$. Fig. $13 \mathrm{~b}$ clearly shows that $\beta_{\text {cobl }}$ follows the director rotation and saturates approaching the same $45^{\circ}$ angle; thus, at large magnetic fields, the FD stripes are parallel to the (average) director, just as in the case of $B_{\mathrm{obl}}=0$.

\subsection{The perpendicular geometry}

Micrographs of flexodomains of the perpendicular geometry recorded slightly above their critical voltage at $B_{\perp}=0 \mathrm{~T}(U=24$ $\mathrm{V}), B_{\perp}=0.2 \mathrm{~T}(U=22.4 \mathrm{~V}), B_{\perp}=0.35 \mathrm{~T}(U=18.4 \mathrm{~V}), B_{\perp}=0.5 \mathrm{~T}$

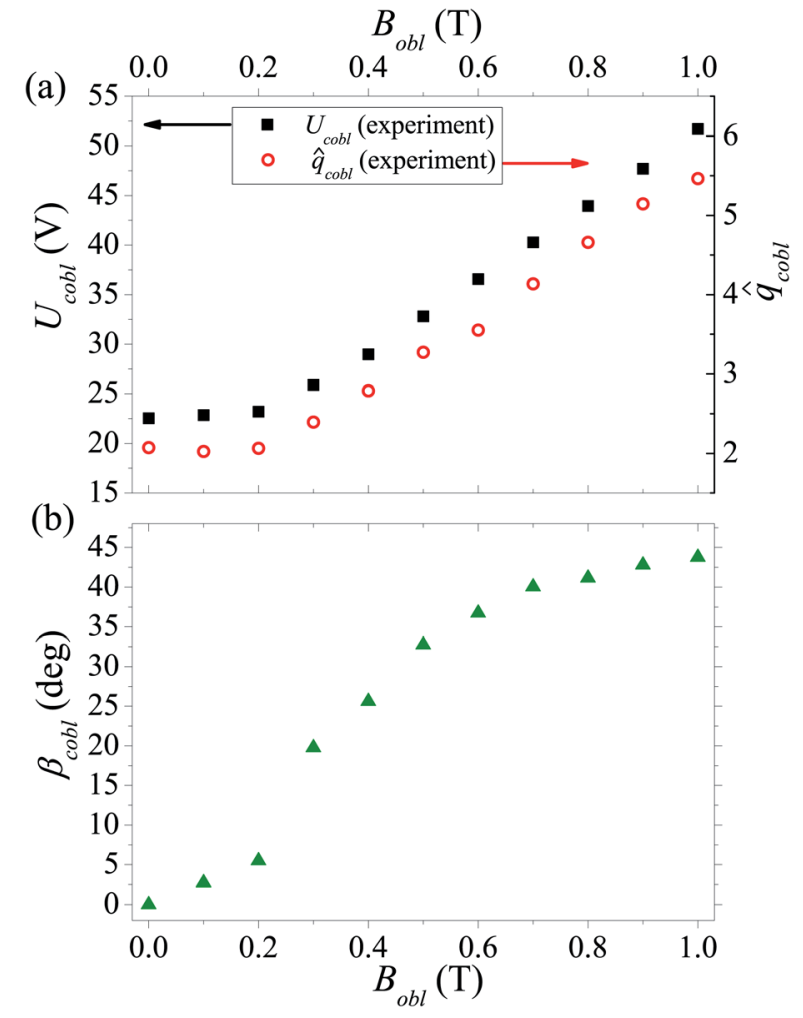

Fig. 13 The magnetic inductance dependence of (a) the critical voltage, wavenumber and (b) FD stripe angle with respect to the rubbing direction in the oblique geometry.

$(U=31.6 \mathrm{~V}), B_{\perp}=0.75 \mathrm{~T}(U=45.6 \mathrm{~V})$ and $B_{\perp}=1 \mathrm{~T}(U=56 \mathrm{~V})$ are shown in Fig. 14a-f, respectively. All images cover an area of $106 \mu \mathrm{m} \times 106 \mu \mathrm{m}$ (the same as shown in Fig. 7 and 11). The direction of the rubbing $\left(\mathbf{n}_{0}\right)$ and that of the magnetic field $\left(B_{\perp}\right)$ correspond to the vertical and the horizontal directions, respectively.

One can observe in Fig. 14a-c that the distance between stripes increases, but the orientation of flexodomains remains parallel to the rubbing direction. In contrast, in Fig. $14 \mathrm{~d}-\mathrm{f}$, the wavenumber of the pattern increases and the stripes become oblique, gradually approaching to be horizontal.

The critical parameters $U_{\mathrm{c} \perp}, \hat{q}_{\mathrm{c} \perp}$, and $\beta_{\mathrm{c} \perp}$ versus $B_{\perp}$ were determined by following the same procedure presented in the previous subsections. The experimental values of the critical voltage, wavenumber and stripe angle can be seen as the function of $B_{\perp}$ in Fig. 15a-c, respectively (solid symbols).

The threshold parameters $U_{\mathrm{c} \perp}, \hat{q}_{\mathrm{c} \perp}$, and $\beta_{\mathrm{c} \perp}$ were also determined by the simulation technique described in Section 3.2. The same material constants were used as for the parallel geometry, except $e^{*}$. A slightly different value of $e^{*}=6.8 \mathrm{pC} \mathrm{m}^{-1}$ was used here (instead of $6.9 \mathrm{pC} \mathrm{m}^{-1}$ ), in order to fit the experimental value of the critical wavenumber in the perpendicular geometry at zero magnetic field, which differed slightly from that measured previously in the parallel geometry. The open symbols in Fig. 15a-c show the magnetic inductance dependence of the critical voltage, wavenumber and stripe angle obtained from the simulations (the connecting lines are just guides for the eye). 
(a)

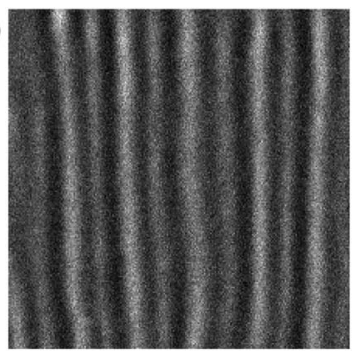

(c)

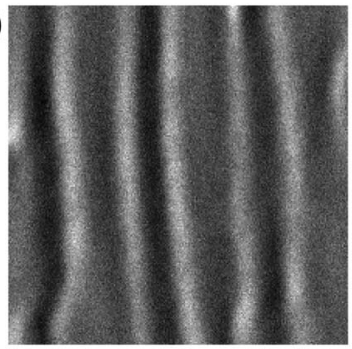

(e)

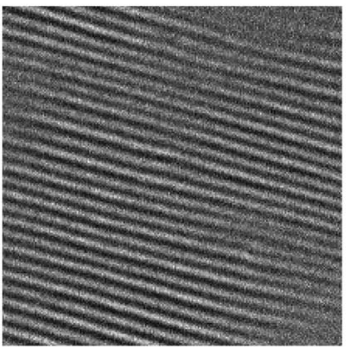

(b)

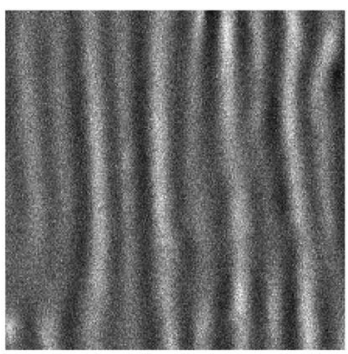

(d)

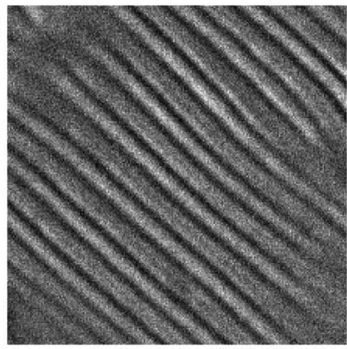

(f)

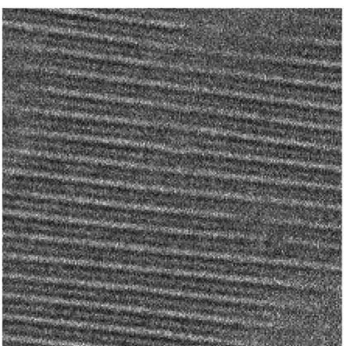

Fig. 14 Micrographs of flexodomains taken at (a) $B_{\perp}=0 \mathrm{~T}(U=24 \mathrm{~V})$, (b) $B_{\perp}=0.2 \mathrm{~T}(U=22.4 \mathrm{~V})$, (c) $B_{\perp}=0.35 \mathrm{~T}(U=18.4 \mathrm{~V})$, (d) $B_{\perp}=0.5 \mathrm{~T}$ $(U=31.6 \mathrm{~V}),(\mathrm{e}) B_{\perp}=0.75 \mathrm{~T}(U=45.6 \mathrm{~V})$ and (f) $B_{\perp}=1 \mathrm{~T}$ (and $\left.U=56 \mathrm{~V}\right)$ in the perpendicular geometry. The images cover an area of $106 \mu \mathrm{m} \times$ $106 \mu \mathrm{m}$. The magnetic field and the rubbing direction lie parallel to the horizontal and the vertical directions, respectively.

It is clear from Fig. 15 that the characteristics of the flexoelectric patterns are different below and above the threshold magnetic inductance $\left(B_{\mathrm{t}}=0.36 \mathrm{~T}\right)$ of the twist Freedericksz transition. Nevertheless, for both $B_{\perp}$ ranges, the theoretical curves nicely reproduce the experimental dependence. In the range $B_{\perp} / B_{\mathrm{t}}<1, U_{\mathrm{c} \perp}$ and $\hat{q}_{\mathrm{c} \perp}$ decreases with increasing magnetic field, while the direction of the FD stripes remains parallel to $\mathbf{n}_{0}$, thus $\beta_{\mathrm{c} \perp}$ is essentially zero. It is important to note that close to $B_{\mathrm{t}}$, both $U_{\mathrm{c} \perp}$ and $\hat{q}_{\mathrm{c} \perp}$ are far below their values at $B_{\perp}=0$. This decrease becomes clear if we invoke the structure of flexodomains. The periodic director deformation of FDs involves both tilt and twist components, as it was described by eqn (12) and (13). The torque exerted by a bias magnetic field applied perpendicular to the initial director helps to twist the director and thus reduces the threshold voltage of FDs, even if it is still too low to induce a homogeneous twist deformation by itself.

Above the Freedericksz threshold, both $U_{\mathrm{c} \perp}$ and $\hat{q}_{\mathrm{c} \perp}$ increase with $B_{\perp}$. Furthermore, the orientation of the stripes changes gradually from the rubbing direction towards a state where they are more parallel to the magnetic field. Therefore, $\beta_{\mathrm{c} \perp}$ increases from zero and approaches $90^{\circ}$ at high values of $B_{\perp}$. In order to see how the critical rotation angle $\beta_{\mathrm{c} \perp}$ of FDs is related to the
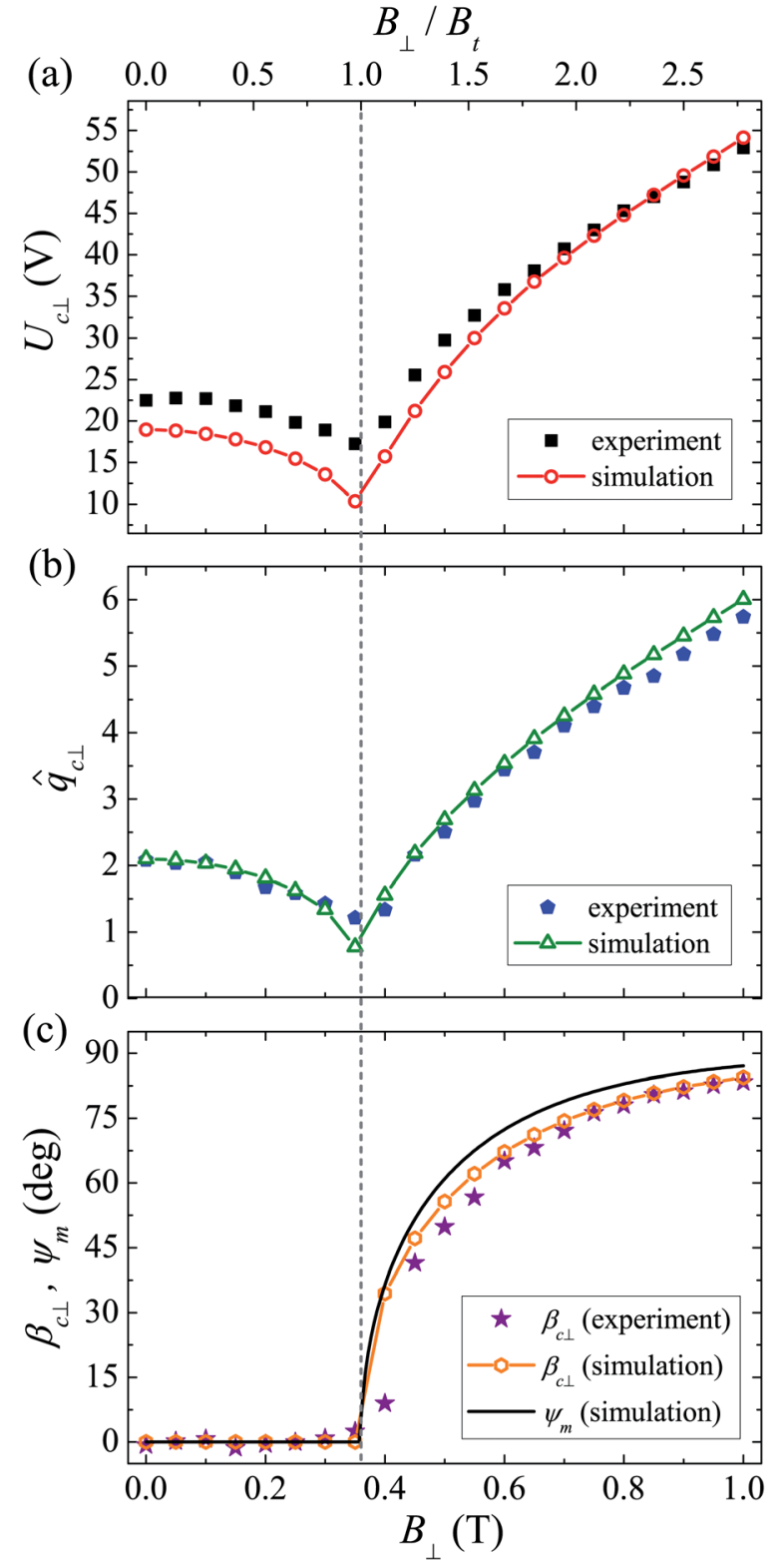

Fig. 15 The magnetic inductance dependence of (a) the critical voltage, (b) wavenumber and (c) FD stripe angle with respect to the rubbing direction in the perpendicular geometry. The solid (connected open) symbols were obtained by experiments (numerical simulations).

twist deformed basic state of the director field, we included the maximal twist angle $\psi_{\mathrm{m}}$ as the function of $B_{\perp}$ in Fig. 15c (solid line). It is clearly seen that $\psi_{\mathrm{m}}$ is always larger than $\beta_{\mathrm{c} \perp}$, as expected. The difference between the stripe angle and the maximal twist angle is relatively small, despite the fact that the $\psi(\hat{z})$ profile is not flat, even at $B_{\perp}=1 \mathrm{~T}$. This leads to the conclusion that the flexoelectric domains in our system are localized in the middle of the cell. As a consequence, the twist deformation of the director is directly visualized by the rotation of the FD stripes.

Though the numerically obtained $U_{\mathrm{c} \perp}\left(B_{\perp}\right)$ curves exhibit a similar $B$-dependence as the experimental ones, the latter values are slightly, though systematically higher, just as has 
been found in the parallel geometry. The explanation given for the voltage deviation in Section 4.1 applies here as well. In contrast to the threshold voltages, the measured and calculated $\hat{q}_{\mathrm{c} \perp}\left(B_{\perp}\right)$ curves match almost perfectly, despite the fact that the $B$-dependent values were not obtained by a fit (no free parameters were varied in the simulations).

Similarly, good agreement can be seen between the calculated and the measured $\beta_{\mathrm{c} \perp}\left(B_{\perp}\right)$ dependence as well, though the threshold of the twist Freedericksz transition seems to be less sharp in the experiment. This is most likely due to experimental imperfections, e.g. a slight misalignment of the magnetic field direction.

We note here that the vertical dashed line in Fig. 15a-c is not an experimental value of the threshold of the twist Freedericksz transition; it was calculated from the material parameters of 1008 listed in Section 2, which were taken from independent measurements. ${ }^{37}$

\section{Discussion}

We have shown in the previous sections that the presence of an additional magnetic field has a significant influence on the formation of flexodomains in a nematic liquid crystal. It is well known that a magnetic field affects the electric Freedericksz transitions as well in certain geometries. This is not surprising as, depending on its direction, the torque exerted by the magnetic field either stabilizes or destabilizes the initial state. In the following, we discuss these analogies in more detail.

Let us start with the magnetic field applied along $\mathbf{n}_{0}$. Here, the critical voltage $U_{\mathrm{c} \|}$ of FDs was found to increase monotonically with $B_{\|}$. Qualitatively similar behavior is expected in the same geometry for the homogeneous splay Freedericksz transition, assuming that the nematic compound exhibits positive dielectric and magnetic susceptibility anisotropies. There, the magnetic field has a stabilizing effect: it tends to suppress the director tilt and twist as well. Our findings point out that this stabilizing effect works similarly in the case of flexodomains, where the director deformation is induced by flexoelectricity, thus acting against the negative dielectric anisotropy that stabilizes the homogeneous planar state.

The perpendicular geometry has some more interesting aspects. Our results show that the critical properties of FDs exhibit a completely different nature in the two distinct magnetic field ranges separated by the twist Freedericksz threshold field. For $B_{\perp}<B_{\mathrm{t}}$, the critical voltage was found to decrease with $B_{\perp}$, while for $B_{\perp}>B_{\mathrm{t}}$ an opposite tendency was detected. Comparing this with the splay Freedericksz transition of a nematic with $\varepsilon_{\mathrm{a}}>0$, we do not find an analogy, in contrast to the parallel geometry. The threshold voltage for the homogeneous director reorientation is not affected by the magnetic field at all if $B_{\perp}<B_{\mathrm{t}}{ }^{44}$ This is due to the fact that the electric splay Freedericksz distortion involves only the tilt of the director, while in FDs tilt and twist are both present. A magnetic inductance below $B_{\mathrm{t}}$ cannot create twist, but may alter twist if it is already present.

Measurements in the oblique and perpendicular geometries showed that the direction of the FD stripes rotate if there is a twist deformation in the sample. This unambiguously proves that the FDs observed are of bulk origin, just as it was assumed in the first theoretical interpretation. ${ }^{\mathbf{1 6}}$ Thus, our findings are in contrast to some recent results of flexoelectric pattern formation in bent-core nematic compounds using twisted cells, ${ }^{24,25}$ where the patterns were found to be localized near the electrodes and changed their direction upon reversal of the voltage polarity. Despite similarities in their appearance, we assume that those patterns are flexodomains of another type with a different, not yet fully explored formation mechanism, where surface effects (e.g. anchoring and ion blocking strength, surface polarization, large electric field gradients near Debye layers) as well as differences in material parameters (ion concentration, elastic constants, etc.) may play an important role.

In Section 1 we have already pointed out the advantages of using flexodomains in determining $e^{*}$, as well as the main drawback of this technique: only a few compounds possess the combination of the material parameters required for the appearance of flexodomains. ${ }^{18}$ For example, in compounds with large positive dielectric anisotropy, FDs cannot be seen because their threshold voltage would be larger than that of the electric Freedericksz transition. However, we showed that applying a magnetic field in the perpendicular geometry substantially decreases $U_{\mathrm{c} \perp}$, while the electric Freedericksz threshold remains unaffected by $B_{\perp}<B_{\mathrm{t}}$. Therefore, our results opens up a perspective to enlarge the number of nematics that may show FDs. Namely, we think that the application of a suitable $B_{\perp}$ will allow observation of FDs in compounds where no flexoelectric pattern formation can be seen in the absence of a magnetic field. Proving this will be the subject of further studies.

\section{Acknowledgements}

Financial support by the Hungarian Research Fund OTKA K81250 and the DAAD/MÖB researcher exchange program (grant no. 29480) are gratefully acknowledged.

\section{References}

1 P. G. de Gennes and J. Prost, The Physics of Liquid Crystals, Clarendon Press, Oxford, 2nd edn, 1993.

2 L. M. Blinov and V. G. Chigrinov, Electrooptic Effects in Liquid Crystal Materials, Springer, New York, 1996.

3 R. Stannarius, in Handbook of Liquid Crystals, ed. J. W. Goodby, P. J. Collings, T. Kato, C. Tschierske, H. Gleeson and P. Raynes, Wiley-VCH, Weinheim, 2014, vol. 3, p. 131.

4 R. B. Meyer, Piezoelectric effects in liquid crystals, Phys. Rev. Lett., 1969, 22, 918-921.

5 Flexoelectricity in Liquid Crystals. Theory, Experiments and Applications, ed. Á. Buka and N. Éber, Imperial College Press, London, 2012.

6 J. Harden, B. Mbanga, N. Éber, K. Fodor-Csorba, S. Sprunt, J. T. Gleeson and A. Jákli, Phys. Rev. Lett., 2006, 97, 157802.

7 J. Harden, R. Teeling, J. T. Gleeson, S. Sprunt and A. Jákli, Phys. Rev. E: Stat., Nonlinear, Soft Matter Phys., 2008, 78, 031702. 
8 P. Sathyanarayana, S. Radhika, B. K. Sadashiva and S. Dhara, Soft Matter, 2012, 8, 2322.

9 W. Weissflog, U. Baumeister, M.-G. Tamba, G. Pelzl, H. Kresse, R. Friedemann, G. Hempel, R. Kurz, M. Roos, K. Merzweiler, A. Jákli, C. Zhang, N. Diorio, R. Stannarius, A. Eremin and U. Kornek, Soft Matter, 2012, 8, 2671.

10 A. Eremin and A. Jákli, Soft Matter, 2013, 9, 615.

11 N. Avci, V. Borshch, D. D. Sarkar, R. Deb, G. Venkatesh, T. Turiv, S. V. Shiyanovskii, N. V. S. Rao and O. D. Lavrentovich, Soft Matter, 2013, 9, 1066.

12 S. Chakraborty, J. T. Gleeson, A. Jákli and S. Sprunt, Soft Matter, 2013, 9, 1817.

13 F. Vita, I. F. Placentino, C. Ferrero, G. Singh, E. T. Samulski and O. Francescangeli, Soft Matter, 2013, 9, 6475.

14 J.-H. Lee, T.-H. Yoon and E.-J. Choi, Soft Matter, 2012, 8, 2370. 15 Pattern Formation in Liquid Crystals, ed. Á. Buka and L. Kramer, Springer, New York 1996.

16 Y. P. Bobylev and S. A. Pikin, Zh. Eksp. Teor. Fiz., 1977, 72, 369; Sov. Phys. JETP, 1977, 45, 195-198.

17 M. I. Barnik, L. M. Blinov, A. N. Trufanov and B. A. Umanski, J. Phys., 1978, 39, 417.

18 A. Krekhov, W. Pesch and Á. Buka, Phys. Rev. E: Stat., Nonlinear, Soft Matter Phys., 2011, 83, 051706.

19 G. Derfel and M. Buczkowska, Mol. Cryst. Liq. Cryst., 2011, 547, 213.

20 F. Lonberg and R. B. Meyer, Phys. Rev. Lett., 1985, 55, 718.

21 P. Tadapatri and K. S. Krishnamurthy, J. Phys. Chem. B, 2012, 116, 782.

22 P. Tadapatri, K. S. Krishnamurthy and W. Weissflog, Soft Matter, 2012, 8, 1202.

23 B. A. Umanski, V. G. Chigrinov, L. M. Blinov and Y. B. Podyachev, Zh. Eksp. Teor. Fiz., 1981, 81, 1307; Sov. Phys. JETP, 1981, 54, 694.

24 K. S. Krishnamurthy, P. Kumar and M. V. Kumar, Phys. Rev. E: Stat., Nonlinear, Soft Matter Phys., 2013, 87, 022504.

25 O. A. Elamain, Bent Core Nematics, Alignment and ElectroOptic Effects, PhD thesis, University of Gothenburg, 2013.

26 K. S. Krishnamurthy, P. Kumar and P. Tadapatri, J. Indian Inst. Sci., 2009, 89, 255.
27 S. L. Cornford, T. S. Taphouse and J. R. Sambles, New J. Phys., 2009, 11, 013045.

28 G. Derfel, J. Mol. Liq., 2009, 144, 59-64.

29 F. C. M. Freire, G. Barbero and M. Scalerandi, Phys. Rev. E: Stat., Nonlinear, Soft Matter Phys., 2006, 73, 051202.

30 F. C. Freire, A. L. Alexe-Ionescu, M. Scalerandi and G. Barbero, Appl. Phys. Lett., 2006, 89, 214101.

31 G. Barbero, A. M. Figueiredo Neto, F. C. M. Freire and J. Le Digabel, Phys. Rev. E: Stat., Nonlinear, Soft Matter Phys, 2006, 74, 052701.

32 G. Barbero, G. Cipparrone, O. G. Martins, P. Pagliusi and A. M. Figueiredo Neto, Appl. Phys. Lett., 2006, 89, 132901.

33 A. L. Alexe-Ionescu, G. Barbero and I. Lelidis, Phys. Rev. E: Stat., Nonlinear, Soft Matter Phys., 2009, 80, 061203.

34 L. O. Palomares, J. A. Reyes and G. Barbero, Phys. Lett. A, 2004, 333, 157-163.

35 R. Atasiei, A. L. Alexe-Ionescu, J. C. Dias, L. R. Evangelista and G. Barbero, Chem. Phys. Lett., 2008, 461, 164169.

36 G. Barbero, F. Batalioto and A. M. Figueiredo Neto, Appl. Phys. Lett., 2008, 92, 172908.

37 P. Salamon, N. Éber, A. Krekhov and Á. Buka, Phys. Rev. E: Stat., Nonlinear, Soft Matter Phys., 2013, 87, 032505.

38 E. Kochowska, S. Németh, G. Pelzl and Á. Buka, Phys. Rev. E: Stat., Nonlinear, Soft Matter Phys., 2004, 70, 011711.

39 G. G. Nair, C. A. Bailey, S. Taushanoff, K. Fodor-Csorba, A. Vajda, Z. Varga, A. Bóta and A. Jákli, Adv. Mater., 2008, 20, 3138.

40 P. Salamon, N. Éber, Á. Buka, J. T. Gleeson, S. Sprunt and A. Jákli, Phys. Rev. E: Stat., Nonlinear, Soft Matter Phys., 2010, 81, 031711.

41 S. Rasenat, G. Hartung, B. L. Winkler and I. Rehberg, Exp. Fluids, 1989, 7, 412.

42 I. Stewart, The Static and Dynamic Continuum Theory of Liquid Crystals, Taylor \& Francis, New York, 2004.

43 N. Éber, L. O. Palomares, P. Salamon, A. Krekhov and Á. Buka, Phys. Rev. E: Stat., Nonlinear, Soft Matter Phys., 2012, 86, 021702.

44 H. J. Deuling, E. Guyon and P. Pieranski, Solid State Commun., 1974, 15, 277. 